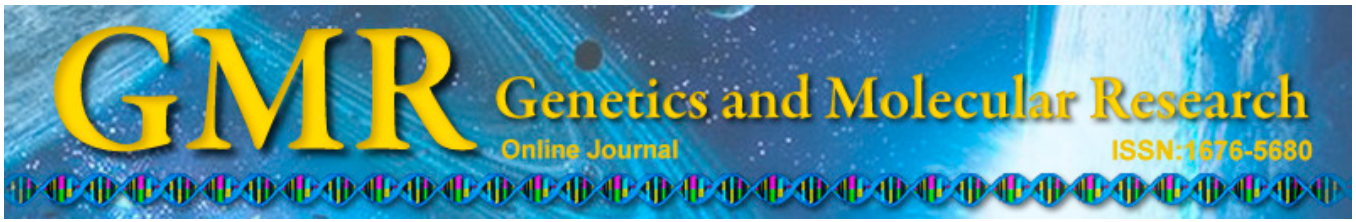

\title{
An expression profiling analysis of hybrid millet and its parents at grain filling stage
}

\author{
Z.H. Liu, H.M. Zhang, G.L. Li, Y.M. Zhang, H.C. Li and X.L. Guo \\ Institute of Genetics and Physiology, \\ Hebei Academy of Agriculture and Forestry Sciences, \\ Plant Genetic Engineering Center of Hebei Province, \\ Shijiazhuang, Hebei Province, China \\ Corresponding author: X.L. Guo \\ E-mail:myhf2002@163.com
}

Genet. Mol. Res. 14 (3): 7821-7832 (2015)

Received November 4, 2014

Accepted March 26, 2015

Published July 14, 2015

DOI http://dx.doi.org/10.4238/2015.July. 14.8

\begin{abstract}
Heterosis has been widely used in crop breeding and production. However, a shortage of genes known to function in heterosis significantly limits our understanding of the molecular basis underlying heterosis. Here, we report 740 differentially expressed genes (DEGs) in the leaves of the hybrid millet Zhang No.5 and its parents at the grain filling stage determined using Solexa Illumina digital gene expression. Of the 740 DEGs, 546 were from the hybrid and its parents and most were up-regulated in the hybrid. Particularly, a large number of DEGs related to starch and carbohydrate metabolism and 2 DEGs encoding chlorophyll $\mathrm{a} / \mathrm{b}$ binding proteins were up-regulated in hybrid millet. Moreover, all DEGs were enriched in the biological process and molecular function, and no DEGs were found to be enriched in the cellular component of GO terms. Pathway enrichment using KEGG showed that several DEGs were enriched in the circadian rhythm pathway. Further analysis revealed that the altered circadian rhythm, which mediates photosynthesis and carbohydrate accumulation, may play an important role in heterosis of the hybrid millet.
\end{abstract}

Key words: Differentially expressed gene; Grain filling stage; Heterosis; Hybrid millet 


\section{INTRODUCTION}

Heterosis is a biological phenomenon in which the offspring from 2 parents show improved and superior performance compared to either inbred parental line. The application of heterosis in agriculture has led to revolutionary increases in the productivity for a number of agricultural species. However, the mechanism of heterosis remains largely unknown. The genes in hybrids are obtained from the parents, and new genes are not introduced. Thus, heterosis may originate from differentially expressed genes (DEGs) (Doebley and Lukens, 1998). Therefore, studies of gene expression levels may reveal the molecular mechanism of heterosis. Great progress has been made in rice and maize. Romagnoli et al. (1990) showed that mRNAs are differentially synthesized and expressed in the $\mathrm{F}_{1}$ primary root tips compared to in parental lines. Xiong et al. (1998) and Wu et al. (2001) found that heterosis was related to inhibition of genes expression in hybrids and not related to expression of the dominant gene. $\mathrm{Ni}$ et al. (2001) showed that over-expressed and hybrid specific expressed genes may play important roles in wheat heterosis. Based on these results, DEGs may be closely related to heterosis.

High-throughput sequencing can greatly improve the efficiency of identifying DEGs, which will provide information to increase the understanding of the molecular basis underlying heterosis. Wei et al. (2009) investigated transcriptome profiles of superhybrid rice LYP9 and its parental cultivars 93-11 and PA64s in 7 tissues and identified 3926 DEGs as a potential group of heterosis-related genes. Song et al. (2010) found 1183 DEGs to be significantly enriched in pathways such as photosynthesis and carbon-fixation, and most key genes involved in the carbon-fixation pathway exhibited up-regulated expression in $F_{1}$ hybrid rice. Qin et al. (2013), provided a repertoire of genes useful for identifying genes involved in maize ear trait heterosis. The large number of genes identified in these studies should be examined to understand the underlying molecular basis of heterosis.

Although foxtail millet (Setaria italica P. Beauv.) is an important cereal crop, hybrid millet was bred successfully until 2000. The yield of hybrid millet was increased greatly (Zhao et al., 2000), but little were known about the heterosis of foxtail millet. Determining the foxtail millet genome sequence may lay a foundation for molecular and biology studies of heterosis (Zhang et al., 2012). In this study, DEGs were identified by high-throughput sequencing to identify a potential group of heterosis-related genes for further study of the mechanism of heterosis in millet.

\section{MATERIAL AND METHODS}

\section{Plant materials}

The hybrid millet Zhang No.5 and parental inbreds were used in this study. The female was a foxtail millet of a photoperiod-sensitive male sterile line. The yield of the hybrid millet was significantly higher than that of the male.

Plants of hybrid millet and its parents were grown in a field. Flag leaves were collected at the beginning of the grain filling stage. All samples were rapidly stored at $-80^{\circ} \mathrm{C}$ until RNA extraction.

RNA samples were isolated from each sample using Trizol reagent (Invitrogen, Carlsbad, CA, USA) according to manufacturer instructions, quantified using a NanoDrop1000 spectrophotometer (Nanodrop Technologies, Wilmington, DE, USA), and labeled. 


\section{Illumina cDNA library preparation and sequencing}

After RNA extraction, mRNAs were purified using oligo-dT-conjugated magnetic beads, and then the mRNA was fragmented into small pieces using divalent cations at high temperature. From these cleaved RNA fragments, 2 cDNA strands were synthesized. A linear polymerase chain reaction (PCR) amplification for 15 cycles was performed for the cDNA to enrich the samples for the desired fragments. The resulting fragments were purified by $2 \%$ certified low-range ultra-pure agarose electrophoresis. After denaturation, single-chain molecules were fixed onto TBS380 (PicoGreen, Life Technologies, Carlsbad, CA, USA). As sequencing template, each molecule developed into a single molecule cluster through in situ amplification. Sequencing by synthesis was performed with a read length of 50 base pairs using the Hiseq2000 (Majorbio, Shanghai, China) according to manufacturer protocols.

\section{Aligning short reads to foxtail millet reference genome}

The original data were evaluated to obtain the clean data using the software SeqPrep (https://github.com/jstjohn/SeqPrep) and sickle (https://github.com/najoshi/sickle). All highquality sequences were mapped to the reference genome from the phytozome web site (http:// www.phytozome.net/foxtailmillet.php).

\section{Screening of DEGs and gene annotation}

A rigorous algorithm was performed to identify DEGs between cultivars. In this study, $\mathrm{P} \leq 0.01$, false discovery rate $\leq 0.05$, and the absolute value of the $\log 2$ Ratio $\geq 1$ (2-fold change) were used as the thresholds to judge the significance of gene expression differences. The results were analyzed using the software Tophat (http://tophat.cbcb.umd.edu/) and Cuffdiff (http://cufflinks.cbcb.umd.edu/). After mapping the reference sequences, the homologs of DEGs with known genes were assigned by homology searching against the National Center for Biotechnology Information Non-Redundancy protein database using BLASTx with an E-value $\leq 1 \mathrm{E}-10$. In addition, Gene Ontology (GO) and pathway enrichment analysis mapped all DEGs in the GO database (http://www.geneontology.org/) into significantly enriched GO terms (Bonferroni corrected P value $\leq 0.05$ ) and DEGs in the Kyoto Encyclopedia of Genes and Genomes database (http://www.kegg.jp/kegg/pathway.html) into metabolic or signal transduction pathways $(\mathrm{P}$ value $\leq 0.05)$ through comparisons with the whole genome background.

\section{Validation of DEGs using real-time-quantitative PCR}

The flag leaves collected at the same time as those used for expression profile analysis but from different plants were used for real-time PCR to validate the DEGs identified by expression profile analysis. A sample of 15 DEGs was selected. Primers were designed using Primer 5 (Table S1) and synthesized by ShengGong Cooperation (Shanghai, China). Total RNA was extracted using TRIzol Reagent (Invitrogen) from approximately $100 \mathrm{mg}$ frozen leaves and treated with DNase I (TaKaRa, Shiga, Japan) to remove potential DNA contamination. Relative quantitative analysis was conducted using the Applied Biosystems 7900HT with SYBR Green PCR Master Mix (Applied Biosystems, Foster City, CA, USA). Three technical replicates were included on each plate. Relative expression levels were computed using the $2^{-\Delta \Delta \mathrm{Ct}}$ method. Two biological replicates were used for real-time PCR analysis. 


\section{RESULTS}

\section{Summary information of DEGs}

Our data were derived from the leaves of 3 cultivars at grain-filling stage. After removing low-quality sequences and trimming adapter sequences, totals of 11,031,026; 9,984,122; and $10,299,335$ high-quality sequences were generated from female, hybrid, and male plants. Next, we blasted these sequences against the genome sequence of foxtail millet (http://www.phytozome.net/ foxtailmillet.php) using the software TopHat (http://tophat.cbcb.umd.edu/). BLAST comparison revealed that $78-81 \%$ of the sequences could be mapped to the foxtail millet genome (Table 1).

Table 1. Summary information of DEGs.
\begin{tabular}{lcccc}
\hline Sample name & $\begin{array}{c}\text { Number of } \\
\text { high-quality sequence }\end{array}$ & $\begin{array}{c}\text { Number of high-quality } \\
\text { base (bp) }\end{array}$ & $\begin{array}{c}\text { Number of } \\
\text { blasting sequence }\end{array}$ & $\begin{array}{c}\text { Percentage of mapping } \\
\text { to the genome (\%) }\end{array}$ \\
\hline Female & $11,031,126$ & $548,195,981$ & $8,698,190$ & 78.85 \\
$\mathrm{~F}_{1}$ & $9,984,122$ & $496,502,744$ & 80.59 \\
male & $10,299,335$ & $511,783,387$ & $8,046,531$ & 81.00 \\
\hline
\end{tabular}

\section{Real-time PCR experiments verified the high-throughput sequencing data}

To evaluate the validity of gene expression analysis using high-throughput sequencing, we performed real-time PCR analysis for 15 genes. The expression based on real-time PCR showed consistent results with those obtained from the sequencing data, demonstrating the satisfactory quality of the sequencing and the data analysis (Table 2).

\begin{tabular}{|c|c|c|c|c|c|}
\hline \multirow[t]{2}{*}{ Gene } & \multirow[t]{2}{*}{ Annotation } & \multicolumn{2}{|c|}{ Sequencing } & \multicolumn{2}{|c|}{ Real-time PCR } \\
\hline & & $\log 2 \mathrm{FC}\left(\mathrm{F}_{1} /\right.$ Female $)$ & $\log 2 \mathrm{FC}\left(\mathrm{F}_{1} / \mathrm{Male}\right)$ & $\mathrm{F}_{1} /$ Female & $\mathrm{F}_{1} /$ Male \\
\hline $\mathrm{Si026463}$ & Chalcone and stilbene synthase family protein & -0.29 & 3.86 & 0.47 & 4.35 \\
\hline Si032777 & Chlorophyll A-B binding family protein & -1.53 & 3.55 & 0.40 & 2.23 \\
\hline $\mathrm{Si031983}$ & UDP-D-glucose/UDP-D-galactose 4-epimerase 1 & -2.15 & -3.90 & 0.47 & 0.41 \\
\hline Si006946 & Peroxidase superfamily protein & 1.77 & 3.93 & 1.23 & 8.34 \\
\hline $\mathrm{Si031242}$ & Basic pathogenesis-related protein 1 & 3.49 & 5.31 & 1.90 & 7.68 \\
\hline Si023769 & Thioredoxin H-type 5 & 4.09 & 4.41 & 2.49 & 31.23 \\
\hline Si000306 & Alpha-glucan phosphorylase 2 & 0.25 & 1.82 & 1.51 & 4.08 \\
\hline Si030429 & WRKY DNA-binding protein 18 & 5.78 & 8.39 & 1.01 & 1.33 \\
\hline Si029769 & UDP-glucosyltransferase $74 \mathrm{~F}_{2}$ & 2.29 & 2.81 & 1.95 & 3.65 \\
\hline $\mathrm{Si} 035705$ & Calreticulin precursor & 2.49 & 2.50 & 1.73 & 4.61 \\
\hline $\mathrm{Si0} 13398$ & MYB family transcription factor & -0.14 & -1.60 & 0.77 & 0.45 \\
\hline Si014685 & MYB family transcription factor & -1.18 & -1.20 & 0.17 & 0.45 \\
\hline Si033968 & Phytochrome B & -0.41 & -1.73 & 0.49 & 0.40 \\
\hline Si033984 & Phytochrome A & -7.06 & -1.87 & 0.32 & 0.33 \\
\hline $\mathrm{Si} 000698$ & Phytochrome interacting factor 3 & -2.10 & -2.74 & 0.51 & 0.65 \\
\hline
\end{tabular}

Positive numbers indicate genes in $F_{1}$ that were up-regulated, and the negative numbers indicate genes in $F_{1}$ that were down-regulated based on the sequencing data; numbers above 1 indicate genes in $F_{1}$ that were up-regulated and numbers less than 1 indicate genes in $\mathrm{F}_{1}$ that were down-regulated from real-time PCR.

\section{Differential expression analysis of hybrid millet and its parents}

According to the BLAST results from all 3 samples, we identified 740 genes significantly differentially expressed in each pairwise comparison (Table S2). Exceeding our 
expectations, the larger number of DEGs (487 DEGs) was found between females and males, while the lowest number of DEGs (177) was found between hybrids and females. A total of 446 DEGs were identified between the hybrid and males. A total of 359 genes, accounting for $48.5 \%$ of all DEGs, appeared to be differentially expressed in more than 1 cultivar, including 11 genes (1.5\% of total DEGs) in 3 cultivars (Figure 1A). Both up- and down-regulation was observed in different cultivars. However, up-regulation dominated in the hybrid, with 132 up-regulated genes (74.6\%) of 177 between the $\mathrm{F}_{1}$ and female and $277(62.1 \%)$ up-regulated genes of 446 between the $F_{1}$ and male. The number of up-regulated genes was 278 of 487 DEGs between females and males (Figure 1B).
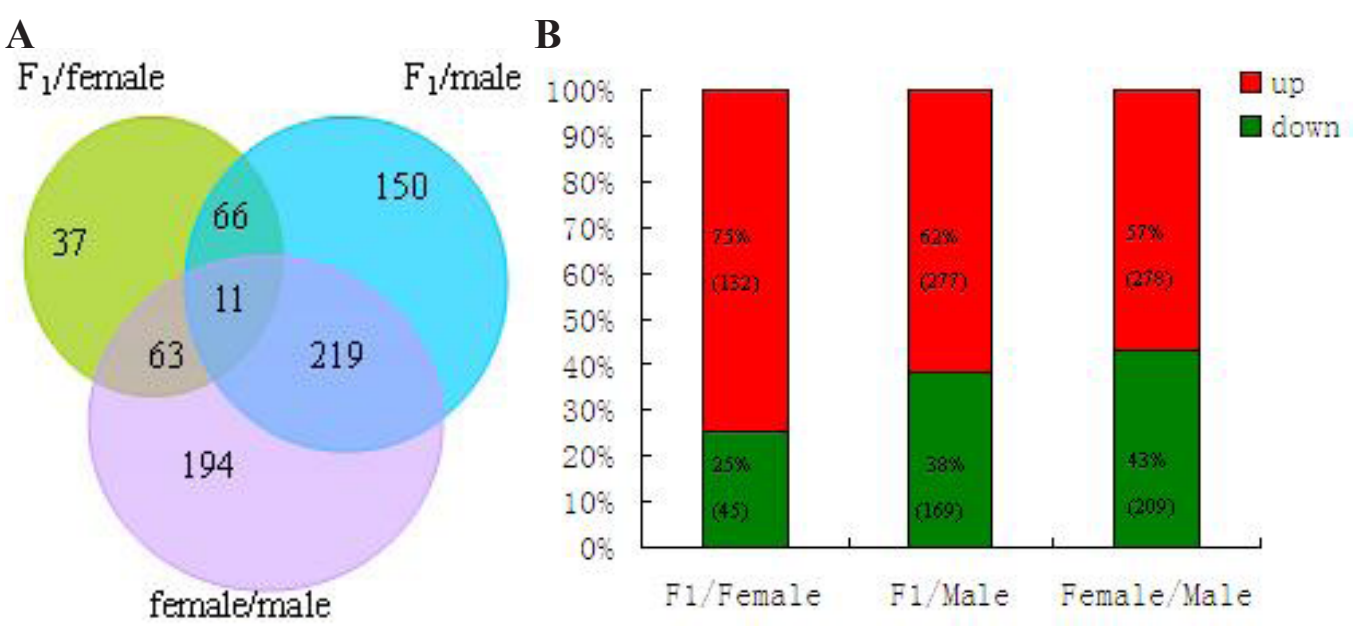

Figure 1. Genes differentially expressed between various cutivars. A. Genes differentially expressed. B. Percentages of up- and down-regulated genes.

\section{GO terms and pathway enrichment of DEGs}

We examined the functional categories of the DEGs using the goatools software (https://github.com/tanghaibao/goatools). We categorized the 740 DEGs according into the general categories of biological process, molecular function, and cellular component (Table 3 ). The DEGs between the hybrid and the female were found in only 5 GO terms. DEGs between hybrid and male were found in $34 \mathrm{GO}$ terms. The number of $\mathrm{GO}$ terms between female and male was largest as the largest number of DEGs were observed between these groups. Although the number of GO terms between cultivars differed, DEGs were all enriched in the biological process and molecular function categories. No DEGs were enriched in the cellular component GO terms, which did not agree with the results that no DEGs between the hybrid maize and its parents were identified in molecular function (Qin et al., 2013). These results suggest that the basis of heterosis differs in different crops. In addition, pathway enrichment analysis was used to analyze potential involvement of genes in cellular metabolic pathways. The DEGs between $F_{1}$ and female were enriched in 6 pathways. Twenty-five DEGs between $F_{1}$ and male were found in the 12 pathways, with some involved in more than 1 pathway. The 
pathways containing the top 3 largest numbers of DEG were phenylpropanoid biosynthesis ( 5 DEGs), antigen processing and presentation (4 DEGs), and amino sugar and nucleotide sugar metabolism (4 DEGs). Furthermore, the 5 DEGs (Si026463, Si033984, Si033968, Si014685, Si013398) were found in the circadian rhythm pathway. Twenty-one DEGs between female and male were found in 12 pathways, 6 of which were shared with the enriched pathway from $\mathrm{F}_{1}$ and male (Table 4).

\section{DEGs involved in circadian rhythms}

The increase in biomass or vitality in $\mathrm{F}_{1}$ is an important aspect of heterosis. A study by $\mathrm{Ni}$ et al. (2009) suggested that the increased biomass or vitality in $\mathrm{F}_{1}$ originated from altered circadian rhythms. In this study, several DEGs involved in the circadian rhythms were identified. Two genes encoding the circadian clock genes CCA1 and LHY (Si014685, Si013398) were down-regulated in hybrids. CCA1 and LHY, which are partially redundant MYB-domain transcription factors with incompletely overlapping functions, were considered to be the central oscillators of the circadian clock (Alabadi et al., 2001; Mizoguchi et al., 2002). The red/ far-red light sensing phytochromes PHYA and PHYB transduce light signals to reset the circadian clock. In this study, the expression of PHYA and PHYB (Si033984, Si033968) were low in the hybrids. Furthermore, the gene (Si000698) encoding the phytochrome-interacting basic helix-loop-helix transcription factor 3 was down-regulated. The expression alteration of DEGs involved in circadian rhythm suggest that these genes play important roles in heterosis.

\section{DEGs involved in carbohydrate metabolism}

Numerous studies have revealed that the metabolism of starch is affected by circadian rhythms ( $\mathrm{Lu}$ et al., 2005; Graf et al., 2010; Shen et al., 2014). Altered circadian rhythms regulate starch accumulation in hybrids and allopolyploids in Arabidopsis (Ni et al., 2009). In this study, a large number of DEGs related to carbohydrate metabolism were identified. The DEGs consisted of the genes related to starch synthesis and the genes responsible for starch degradation, including 3 genes encoding glycosyl hydrolases family 17 (Si002182, Si004560, Si022625), 2 genes encoding amylase (Si021452, Si035044), 2 genes encoding carbohydrate phosphorylase (Si000306, Si034019), 1 gene encoding aldehyde dehydrogenase (Si000743), 3 genes encoding UDP-glucoronosyl/UDP-glucosyl transferase (Si006342, Si029769, Si040038), 2 genes responsible for transporter family protein (Si030126, Si030450), the trehalose synthase gene (Si000238), and the UDP-glucose 4-epimerase gene (Si031983). These genes were all up-regulated in the hybrid except for 2 genes (Si000238, Si031983). These DEGs suggest that carbohydrate metabolism is vigorous in the hybrid. The up-regulation of glucosyl transferase indicates that starch metabolism in the hybrid is particularly vigorous.

\section{DEGs involved in photosynthesis}

Hybrids nearly always show higher photosynthetic capacity, which has been observed in Arabidopsis hybrids (Fujimoto et al., 2012), super-hybrid rice (Wang et al., 2000; Song et al., 2010), maize hybrids (Tollenaar et al., 2004), and hybrid wheat (Yang et al., 2007). Similarly, hybrid millet showed higher photosynthetic capacity because of the higher chlorophyll 
Table 3. Significant GO terms of DEGs in the GO annotation analysis (false-discovery rate-corrected P values, 0.05 ).

\begin{tabular}{|c|c|c|}
\hline GO Term & Description & $P_{-}$fdr \\
\hline \multicolumn{3}{|l|}{$\mathrm{F} /$ Female } \\
\hline GO:0009607 & Response to biotic stimulus & 0.015 \\
\hline GO:0006952 & Defense response & 0.033 \\
\hline GO:0005488 & Binding & 0.046 \\
\hline GO:0001071 & Nucleic acid binding transcription factor activity & 0.046 \\
\hline GO:0003700 & Sequence-specific DNA binding transcription factor activity & 0.046 \\
\hline \multicolumn{3}{|c|}{ Seque } \\
\hline GO:0043531 & ADP binding & 0 \\
\hline GO:0008219 & Cell death & 0 \\
\hline GO:0016265 & Death & 0 \\
\hline GO:0012501 & Programmed cell death & 0 \\
\hline GO:0006915 & Apoptotic process & 0 \\
\hline GO:0043168 & Anion binding & 0 \\
\hline GO:0032553 & Ribonucleotide binding & 0 \\
\hline GO:0000166 & Nucleotide binding & 0 \\
\hline GO: 1901265 & Nucleoside phosphate binding & 0 \\
\hline GO:0036094 & Small molecule binding & 0 \\
\hline GO: 1901363 & Heterocyclic compound binding & 0 \\
\hline GO:0097159 & Organic cyclic compound binding & 0 \\
\hline GO:0032555 & Purine ribonucleotide binding & 0 \\
\hline GO:0032550 & Purine ribonucleoside binding & 0 \\
\hline GO:0001883 & Purine nucleoside binding & 0 \\
\hline GO:0035639 & Purine ribonucleoside triphosphate binding & 0 \\
\hline GO:0017076 & Purine nucleotide binding & 0 \\
\hline GO:0032549 & Ribonucleoside binding & 0 \\
\hline GO:0001882 & Nucleoside binding & 0 \\
\hline GO:0032559 & Adenyl ribonucleotide binding & 0 \\
\hline GO:0005524 & ATP binding & 0 \\
\hline GO:0030554 & Adenyl nucleotide binding & 0 \\
\hline GO:0043167 & Ion binding & 0 \\
\hline GO:0009987 & Cellular process & 0 \\
\hline GO:0003674 & Molecular function & 0 \\
\hline GO:0008150 & Biological process & 0 \\
\hline GO:0005488 & Binding & 0 \\
\hline GO:0005509 & Calcium ion binding & 0.002 \\
\hline GO:0009055 & Electron carrier activity & 0.005 \\
\hline GO:0020037 & Heme binding & 0.011 \\
\hline GO:0016491 & Oxidoreductase activity & 0.011 \\
\hline GO:0046906 & Tetrapyrrole binding & 0.012 \\
\hline GO:0046872 & Metal ion binding & 0.012 \\
\hline GO:0065008 & Regulation of biological quality & 0.017 \\
\hline GO:0008152 & Metabolic process & 0.017 \\
\hline GO:0019725 & Cellular homeostasis & 0.017 \\
\hline GO:0042592 & Homeostatic process & 0.018 \\
\hline GO:0003824 & Catalytic activity & 0.024 \\
\hline GO:0005515 & Protein binding & 0.024 \\
\hline GO:0044710 & Single-organism metabolic process & 0.03 \\
\hline \multicolumn{3}{|l|}{$\mathrm{F}_{1} / \mathrm{Male}$} \\
\hline GO:0008219 & Cell death & 0 \\
\hline GO:0016265 & Death & 0 \\
\hline GO:0043531 & ADP binding & 0 \\
\hline GO:0012501 & Programmed cell death & 0 \\
\hline GO:0006915 & Apoptotic process & 0 \\
\hline GO:0032559 & Adenyl ribonucleotide binding & 0 \\
\hline GO:0005524 & ATP binding & 0 \\
\hline GO:0030554 & Adenyl nucleotide binding & 0 \\
\hline GO:0032553 & Ribonucleotide binding & 0 \\
\hline GO:0008150 & Biological process & 0 \\
\hline GO:0032555 & Purine ribonucleotide binding & 0 \\
\hline GO:0032550 & Purine ribonucleoside binding & 0 \\
\hline GO:0001883 & Purine nucleoside binding & 0 \\
\hline GO:0035639 & Purine ribonucleoside triphosphate binding & 0 \\
\hline
\end{tabular}

Continued on next page 
Table 3. Continued.

\begin{tabular}{|c|c|c|}
\hline GO Term & Description & P_fdr \\
\hline GO:0017076 & Purine nucleotide binding & 0 \\
\hline GO:0032549 & Ribonucleoside binding & 0 \\
\hline GO:0001882 & Nucleoside binding & 0 \\
\hline GO:1901363 & Heterocyclic compound binding & 0 \\
\hline GO:0097159 & Organic cyclic compound binding & 0 \\
\hline GO:0003674 & Molecular function & 0 \\
\hline GO:0043168 & Anion binding & 0 \\
\hline GO:0043167 & Ion binding & 0 \\
\hline GO:0036094 & Small molecule binding & 0 \\
\hline GO:0000166 & Nucleotide binding & 0 \\
\hline GO:1901265 & Nucleoside phosphate binding & 0 \\
\hline GO:0009055 & Electron carrier activity & 0 \\
\hline GO:0016705 & Oxidoreductase activity, acting on paired donors, with incorporation or reduction of molecular oxygen & 0.002 \\
\hline GO:0005488 & Binding & 0.002 \\
\hline GO:0009987 & Cellular process & 0.002 \\
\hline GO:0003824 & Catalytic activity & 0.003 \\
\hline GO:0044424 & Intracellular part & 0.007 \\
\hline GO:0020037 & Heme binding & 0.007 \\
\hline GO:0046906 & Tetrapyrrole binding & 0.007 \\
\hline GO:0004645 & Phosphorylase activity & 0.031 \\
\hline
\end{tabular}

Table 4. Enriched pathways for DEGs.

\begin{tabular}{|c|c|c|c|}
\hline Pathway term & Sample No. & Genes & $P$ value \\
\hline \multicolumn{4}{|l|}{$\mathrm{F}_{1} /$ female } \\
\hline DDT degradation & 1 & $\mathrm{Si} 004534$ & 0.019218 \\
\hline Chlorocyclohexane and chlorobenzene degradation & 1 & $\mathrm{Si} 004534$ & 0.023018 \\
\hline Novobiocin biosynthesis & 1 & $\mathrm{Si} 017185$ & 0.030577 \\
\hline Cysteine and methionine metabolism & 2 & Si017185, Si001369 & 0.036295 \\
\hline Phenylpropanoid biosynthesis & 2 & Si013626, Si009896 & 0.042438 \\
\hline Tropane, piperidine and pyridine alkaloid biosynthesis & 1 & $\mathrm{Si} 017185$ & 0.049226 \\
\hline \multicolumn{4}{|l|}{$\mathrm{F}_{1} /$ male } \\
\hline Antigen processing and presentation & 4 & Si021434, Si000619, Si035705, Si001225 & $4.28 \mathrm{E}-05$ \\
\hline Flavonoid biosynthesis & 3 & Si035224, Si001126, Si026463 & 0.000641 \\
\hline Phenylpropanoid biosynthesis & 5 & Si001126, Si000743, Si016467, Si013626, Si030593 & 0.001335 \\
\hline Legionellosis & 3 & $\mathrm{Si} 021434, \mathrm{Si} 034887, \mathrm{Si} 000619$ & 0.005765 \\
\hline Circadian rhythm-plant & 5 & Si026463, Si033984, Si033968, Si014685, Si013398 & 0.00666 \\
\hline Fatty acid biosynthesis & 2 & Si006496, Si010296 & 0.011449 \\
\hline Amino sugar and nucleotide sugar metabolism & 4 & Si035310, Si021771, Si031983, Si008781 & 0.012824 \\
\hline Measles & 2 & Si021434, Si000619 & 0.022244 \\
\hline MAPK signaling pathway & 2 & Si021434, Si000619 & 0.024029 \\
\hline Phenylalanine metabolism & 3 & Si001126, Si016467, Si030593 & 0.027878 \\
\hline Toxoplasmosis & 2 & Si021434, Si000619 & 0.038044 \\
\hline Nitrogen metabolism & 2 & $\mathrm{Si} 021521, \mathrm{Si} 016467$ & 0.038044 \\
\hline \multicolumn{4}{|l|}{ Female/male } \\
\hline Nitrogen metabolism & 4 & Si021521, Si016467, Si013224, Si016715 & 0.001408 \\
\hline Flavonoid biosynthesis & 3 & $\mathrm{Si035224,} \mathrm{Si026463,} \mathrm{Si021789}$ & 0.002496 \\
\hline Antigen processing and presentation & 3 & Si021434, Si000619, Si009497 & 0.003932 \\
\hline Naphthalene degradation & 2 & $\mathrm{Si} 036012, \mathrm{Si} 026503$ & 0.006268 \\
\hline Retinol metabolism & 2 & Si036012, Si026503 & 0.009872 \\
\hline Metabolism of xenobiotics by cytochrome P450 & 2 & $\mathrm{Si} 036012, \mathrm{Si} 026503$ & 0.009872 \\
\hline Phenylpropanoid biosynthesis & 5 & Si016467,Si000743, Si021789, Si009896, Si030593 & 0.010047 \\
\hline Chloroalkane and chloroalkene degradation & 2 & $\mathrm{Si} 036012, \mathrm{Si} 026503$ & 0.014189 \\
\hline Drug metabolism - cytochrome P450 & 2 & $\mathrm{Si} 036012, \mathrm{Si} 026503$ & 0.014189 \\
\hline Porphyrin and chlorophyll metabolism & 3 & Si010922, Si026773, Si001132 & 0.017846 \\
\hline Legionellosis & 3 & Si021434, Si034887, Si000619 & 0.020622 \\
\hline Fatty acid biosynthesis & 2 & Si006496, Si010296 & 0.027804 \\
\hline
\end{tabular}


$\mathrm{a} / \mathrm{b}$ value compared with that of conventional millet (Liu et al., 2012). In this study, 2 genes (Si031233, Si032777) encoding chlorophyll a/b binding proteins were up-regulated, which is significant for photosynthesis. Qin et al. (2013) identified 4 DEGs encoding chlorophyll a/b binding proteins in the developing top ear shoots between a maize heterotic $\mathrm{F}_{1}$ hybrid (Mo179 $x$ B73) and its parental inbreds using maize microarrays. These results suggest that the differential expression of chlorophyll $\mathrm{a} / \mathrm{b}$ may play an important role in the higher photosynthetic capacity of the hybrid. In addition, the expression of 2 genes (Si011039, Si026463) encoding chalcone synthase were increased in the hybrid, which is important for protecting photosynthetic organs against ultraviolet light (Chappell and Hahlbrock, 1984). This protection may be another important factor for the increased photosynthetic capacity.

\section{DISCUSSION}

Differential gene expression underlies the molecular basis of differential trait heterosis. However, nearly all traits exhibiting heterosis are quantitatively inherited and controlled by numerous genes. High-throughput sequencing enables identification of a large number of genes, which will increase the understanding of the heterosis mechanism. Genome-wide changes in gene expression have been documented in intraspecific hybrids of Arabidopsis thaliana (Andorf et al., 2010; Fujimoto et al., 2012; Shen et al., 2012), rice (Wei et al., 2009; He et al., 2010, Song et al., 2010), maize (Swanson-Wagner et al., 2006; Guo et al., 2006; Stupar and Spinger, 2006), and wheat (Wang et al., 2006), and in allopolyploids of Arabidopsis (Wang et al., 2006; Shi et al., 2012) and wheat (Qi et al., 2012). Nevertheless, heterosis-related DEGs and/or their regulatory mechanisms vary significantly between species (Qin et al., 2013). In this study, we identified 740 DEGs between hybrid millet and its parent lines. Most of these genes were up-regulated in the hybrid. Furthermore, nearly all of the genes have been functionally annotated. Therefore, this study provides a repertoire of genes useful for studying the underlying molecular basis of heterosis.

Recent studies have indicated that over $30 \%$ of the $A$. thaliana transcriptome is driven by the circadian clock (Harmer et al., 2000; Covington et al., 2008), thus potentially regulating numerous metabolic pathways (Covington et al., 2008), such as starch metabolism, chlorophyll synthesis, and jasmonate and salicylates accumulation (Danielle et al., 2013), among others. CCA1 and LHY, as key elements of the circadian clock, were down-regulated and induced heterosis in hybrids and allopolyploids (Ni et al., 2009). Our results agreed with those of the previous study. In this study, CCA1 and LHY were down-regulated in hybrid millet. In addition, PHYA and phytochrome-interacting basic helix loop helix transcription factor 3 were down-regulated. PHYA is thought to be the only active photoreceptor that can mediate far-red light input to the circadian rhythm. Down-regulation of PHYA in the hybrid millet may induce down-regulation of CCA1and LHY, which then induce a series of downstream events. The greatest changes induced by the circadian rhythm in this study were the increased metabolism of starch and the chlorophyll because the DEGs (Si031233, Si032777) encoding chlorophyll $\mathrm{a} / \mathrm{b}$ binding proteins and the DEGs related to carbohydrate metabolism were up-regulated. The increased chlorophyll level enhanced the photosynthetic capacity and altered carbohydrate accumulation, resulting in the heterosis of biomass and yield of the hybrid millet (Figure 2). Although the hybrid vigor or heterosis is undoubtedly complex, our findings increase the understanding of heterosis. 


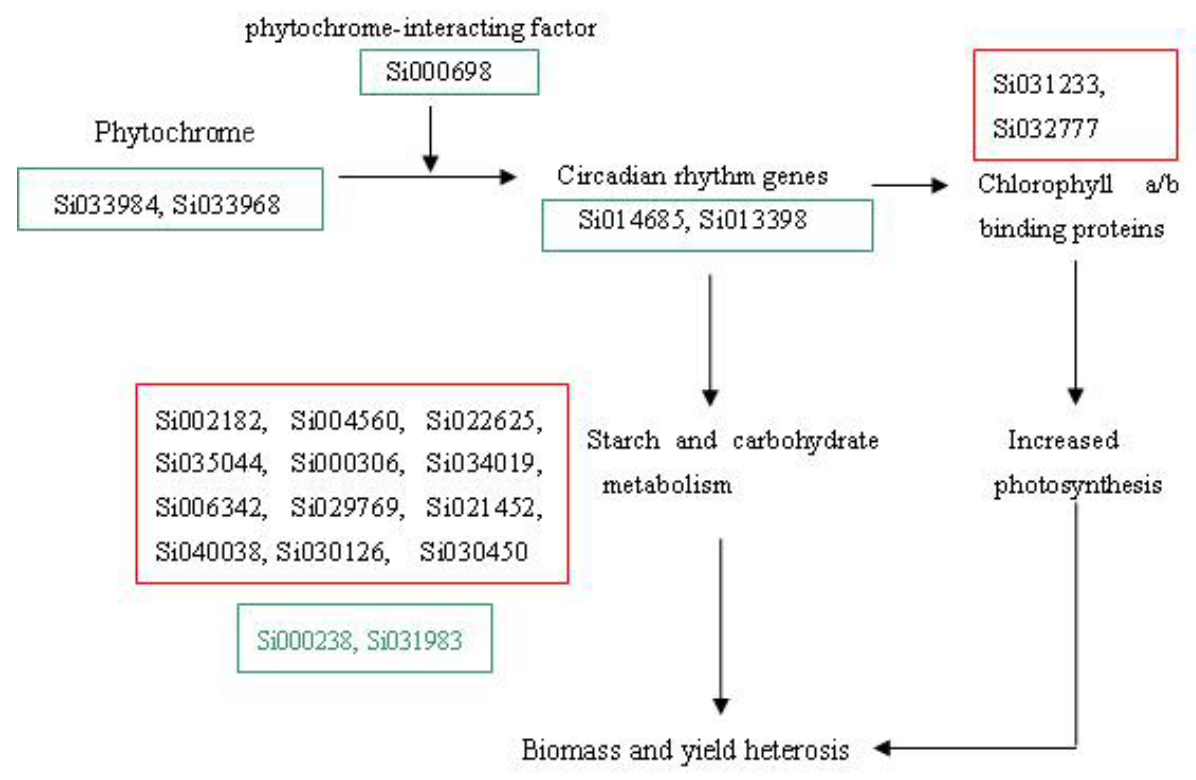

Figure 2. Schematic representation of DEGs involved in the circadian rhythm pathway and the biological process regulated by the circadian clock. The diagram was adapted from 2 papers (Ni et al., 2009; Chen, 2013). The DEGs are indicated in colored rectangular boxes, with red for DEGup and green for DEGdown.

Based on our results, circadian clock genes were clearly related to heterosis. Because light is a key factor in setting the circadian clock, the difference in photoreceptors may induce differential expression of circadian clock genes. At least 3 families of photoreceptors have been identified as transducing light signals to reset the clock, including the blue light-sensing cryptochromes, the red/far-red light sensing phytochromes (PHYA, PHYB, PHYD, PHYE) (Yanovsky et al., 2000), and a family of three F-box proteins, including ZEITLUPE (Baudry et al., 2010). In this study, the down-regulation of circadian clock genes in hybrid millet were induced by red/far-red light sensing the phytochromes (PHYA, PHYB). The characteristics of hybrids may be inherited from the female of a photoperiod-sensitive male sterile line. The difference in expression of genes encoding PHYA and PHYB (Si033984, Si033968) between the hybrid and female was not significant. However, no DEG encoding any phytochromes was found in a study by Song et al. (2010), although circadian clock genes were also downregulated in hybrid rice. This indicates that the causes of differential expression of circadian clock genes are complex. Additionally, the basis of heterosis differs between species.

\section{ACKNOWLEDGMENTS}

Research supported by the National Science and Technology Supporting Plan (\#2011-BAD06B00) and the Key Basic Research Projects of the Basic Research for Application Plan of Hebei Province (\#11965517D).

\section{Supplementary material}




\section{REFERENCES}

Alabadi D, Oyama T, Yanovsky MJ, Haarmon FG, et al. (2001). Reciprocal regulation between TOC1 and LHY/CCA1 within the Arabidopsis circadian clock. Science 293: 880-883.

Andorf S, Selbig J, Altmann T, Poos K, et al. (2010). Enriched partial correlations in genome-wide gene expression profiles of hybrids (A. thaliana): a systems biological approach towards the molecular basis of heterosis. Theor. Appl. Genet. 120: 249-259.

Baudry A, Ito S, Song YH, Strait AA, et al. (2010). F-box proteins FKF1 and LKP2 act in concert with ZEITLUPE to control Arabidopsis clock progression. Plant Cell 22: 606-622.

Chappell J and Hahlbrock K (1984). Transcription of plant defense genes in response to UV light or fungal elicitor. Nature 311: 76-78.

Chen ZJ (2013). Genomic and epigenetic insights into the molecular bases of heterosis. Nat. Rev. Genet. 14: 471-482.

Covington MF, Maloof JN, Straume M, Kay SA, et al. (2008). Global transcriptome analysis reveals circadian regulation of key pathways in plant growth and development. Genome Biol. 9: R130.

Danielle G, Chehab EW, Covington MF and Braam J (2013). Circadian control of jasmonates and salicylates. Plant Signal. Behav. 8: e23123.

Doebley J and Lukens L (1998). Transcriptional regulators and the evolution of plant form. Plant Cell 10: 1075-1082.

Fujimoto R, Taylor JM, Shirasawa S, Peacock WJ et al. (2012). Heterosis of Arabidopsis hybrids between C24 and Col is associated with increased photosynthesis capacity. Proc. Natl. Acad. Sci. U. S. A. 109: 7109-7114.

Graf A, Schlereth A, Stitt M and Smith AM (2010). Circadian control of carbohydrate availability for growth in Arabidopsis plants at night. Proc. Natl. Acad. Sci. U. S. A. 107: 9458-9463.

Guo M, Rupe MA, Yang X, Crasta O, et al. (2006).Genome-wide transcript analysis of maize hybrids: allelic additive gene expression and yield heterosis. Theor. Appl. Genet. 113: 831-845.

Harmer SL, Hogenesch JB, Straume M, Chang HS, et al. (2000). Orchestrated transcription of key pathways in Arabidopsis by the circadian clock. Science 290: 2110-2113.

He G, Zhu X, Elling AA, Chen L, et al. (2010). Global epigenetic and transcriptional trends among two rice subspecies and their reciprocal hybrids. Plant Cell 22: 17-33.

Liu, ZH, Zhang HM, Zhang YM, Li GL, et al. (2012). Photosynthetic characteristics of hybrid millet at grain filling stage. Acta Agric. Boreali-Occidentalis Sin. 21: 60-64.

Lu Y, Gehan JP and Sharkey TD (2005). Daylength and circadian effects on starch degradation and maltose metabolism. Plant Physiol. 138: 2280-2291.

Mizoguchi T, Wheatley K, Hanzawa Y, Wright L, et al. (2002). LHY and CCA1 are partially redundant genes required to maintain circadian rhythms in Arabidopsis. Dev. Cell 2: 629-641.

Ni ZF, Sun QX, Wu LM, Xie CF, et al. (2001). Alternation of gene expression in wheat hybrid $\mathrm{F}_{1}$ and their parental seedling leaves. J. Agric. Biotechnol. 9: 366-370.

Ni ZF, Kim ED, Ha M, Lackey E, et al. (2009). Altered circadian rhythms regulate growth vigour in hybrids and allopolyploids. Nature 457: 327-333.

Qi B, Huang W, Zhu B, Zhong X, et al. (2012). Global transgenerational gene expression dynamics in two newly synthesized allohexaploid wheat (Triticum aestivum) lines. BMC Biol. 10: 3 .

Qin J, Scheuring CF, Wei G, Zhi H, et al. (2013). Identification and characterization of a repertoire of genes differentially expressed in developing top ear shoots between a superior hybrid and its parental inbreds in Zea mays L. Mol. Genet. Genomics 288: 691-705.

Romagnoli S, Maddaloni M, Livini C and Motto M (1990). Relationship between gene expression and hybrid vigor in primary root tips of young maize (Zea mays L.) plantlets. Theor. Appl. Genet. 80: 767-775.

Shen G, Hu W, Zhang B and Xing Y (2014). The regulatory network mediated by circadian clock genes is related to heterosis in rice. J. Integr. Plant Biol. 12240.

Shen H, He H, Li J and Chen W (2012). Genome-wide analysis of DNA methylation and gene expression changes in two Arabidopsis ecotypes and their reciprocal hybrids. Plant Cell 24: 875-892.

Shi X, Ng DW, Zhang C, Comai L, et al. (2012). Cis- and trans-regulatory divergence between progenitor species determines gene expression novelty in Arabidopsis allopolyploids. Nat. Commun. 3: 950

Song GS, Zhai HL, Peng YG and Zhang L (2010). Comparative transcriptional profiling and preliminary study on heterosis mechanism of super-hybrid rice. Mol. Plant 3: 1012-1025.

Stupar RM and Springer NM (2006). Cis-transcriptional variation in maize inbred lines B73 and Mo17 leads to additive expression patterns in the $\mathrm{F}_{1}$ hybrid. Genetics 173: 2199-2210.

Swanson-Wagner RA, Jia Y, DeCook R, Borsuk LA, et al. (2006). All possible modes of gene action are observed in a global comparison of gene expression in a maize $\mathrm{F}_{1}$ hybrid and its inbred parents. Proc. Natl. Acad. Sci. U. S. A. 103: 
6805-6810.

Tollenaar M, Ahmadzadeh A and Lee EA (2004). Physiological basis of heterosis for grain yield in maize. Crop 44: 2086-2094.

Wang J, Tian L, Lee HS, Wei NE, et al. (2006). Genomewide nonadditive gene regulation in Arabidopsis allotetraploids. Genetics 172: 507-517.

Wang Q, Zhang QD, Jiang GM, Lu CM, et al. (2000). Photosynthetic characteristics of two super high-yield hybrid rice. Acta Bot. Sin. 42: 1285-1288.

Wang Z, Ni Z, Wu H, Nie X, et al. (2006). Heterosis in root development and differential gene expression between hybrids and their parental inbreds in wheat (Triticum aestivum L.). Theor. Appl. Genet. 113: 1283-1294.

Wei G, Tao Y, Liu GZ, Chen C, et al. (2009). A transcriptomic analysis of superhybrid rice LYP9 and its parents. Proc. Natl. Acad. Sci. U. S. A. 106: 7695-7701.

Wu MS, Gao ZH and Dai JR (2001). Studies on differential gene expression of maize (Zea mays L.) by means of cDNAAFLP technique. Acta Agronom. Sin. 27: 339-342.

Xiong LZ, Yang GP, Xu CG, Zhang QF, et al. (1998). Relationships of differential gene expression in leaves with heterosis and heterozygosity in a rice diallel cross. Mol. Breed. 4: 129-136.

Yang X, Chen X, Ge Q, Li B, et al. (2007). Characterization of photosynthesis of flag leaves in a wheat hybrid and its parents grown under field conditions. J. Plant Physiol. 164: 318-326.

Yanovsky MJ, Mazzella MA and Casal JJ (2000). A quadruple photoreceptor mutant still keeps track of time. Curr. Biol. 10: 1013-1015.

Zhang G, Liu X, Quan Z, Cheng S et al. (2012). Genome sequence of foxtail millet (Setaria italica) provides insights into grass evolution and biofuel potential. Nat. Biotechnol. 30: 549-554.

Zhao ZH, Xu YS and Zhu XH (2000). The ways and prospects of heterotic utilization of foxtail millet. J. Zhangjiakou Agric. Col. 1: 1-2. 